\title{
La violencia en las relaciones de pareja en estudiantes universitarios. Propuestas educativas
}

\section{The violence in couple's relationship In university students. Educational propositions}

\author{
Andrés Soriano Díaz \\ UNIVERSIDAD DE GRANADA
}

Resumen

En el presente artículo se muestran alguno de los principales resultados obtenidos en la investigación realizada sobre violencia en las relaciones de pareja en el alumnado de $\mathrm{Ma}-$ gisterio en la Facultad de Ciencias de la Educación de la Universidad de Granada. Dicho estudio, de carácter descriptivo, pone de manifiesto las características del fenómeno en relación con la tipología, la incidencia y el grado de autopercepción del problema. Para ello se ha utilizado un cuestionario, elaborado ex profeso, compuesto por veinte ítems agrupados en categorías y organizados en subcategorías, para una mejor diferenciación y análisis, que refleja acciones concretas en las que se manifiestan distintas tipologías de malos tratos a través de una escala que indica la frecuencia e intensidad de los mismos. Los datos obtenidos muestran que el $21,2 \%$ del lumnado encuestado, ha padecido, por parte de su pareja, con frecuencia, alouna forma de violencia de las señaladas en los distintos ítems del cuestionario y cómo estas conductas afectan en mayor proporción a las mujeres que a los hombres. De igual modo se constata el bajóndice de autopercepción que existe del problema en los estudiantes encuestados. Finalmente, se plantea la necesidad de trabajar, desde la formación de los futuros educadores, en la detección y prevención de la violencia en las relaciones de pareja y se recogen algunas propuestas de intervención educativa en este ámbito.

Palabras CLAVE: Violencia, relaciones de pareja, universitarios, prevención, intervención, propuestas educativas.

\section{Summary}

In the present article the main obtained results from a research on violence in couple's relationships are shown. This research was carried out on students from the Faculty of Education of the University of Granada. This study is mainly descriptive and shows the 
characteristics of this phenomenon in relation to the typology, incidence and grade of self-perception of the problem. Obtained data show that $21,2 \%$ of the students have frequently suffered some kind of violence - poin ted out in the questionnaire- from their couples, and how those behaviours affect mainly women than men. Also, the low grade of self-perception of the problem by the students is acknowledged. Finally, the necessity of working, during the education of the future teachers, on the detection and prevention of violence in the couple is suggested and some educative intervention principles in this field are proposed

KEY WORDS: Violence, couple's relationships, university students, prevention, intervention, educative principles.

\section{Introducción}

La expresión violencia en las relaciones de pareja hace referencia a las formas de malos tratos que, ejercidas tanto por hombres como por mujeres, persiguen la consecución de una situación de conformidad y control sobre uno de los miembros de la pareja. Este problema que en la actualidad presenta una creciente incidencia y causa lesiones físicas, psicológicas e, incluso, en algunos casos la muerte, tiene su origen en las relaciones de poder desiguales que se establecen desde el miembro más fuerte hacia el más débil. En la mayor parte de los casos, la violencia es ejercida por los hombres contra las mujeres denominándose violencia de género; sin embargo, el fenómeno es más amplio y abarcativo por lo que para poder afrontarlo con garantías es necesario un conocimiento del mismo en profundidad y en todas sus dimensiones.

En este trabajo presentamos parte de los resultados obtenidos en el proyecto "Violencia en las relaciones de pareja: malos tratos en estudiantes de magisterio en la Facultad de
Ciencias de la Educación de la Universidad de Granada" financiado por el Plan Propio de investigación de la Universidad de Granada ${ }^{1}$. Nuestra investigación, que focaliza su atención en el alumnado universitario, pretende aportar conocimiento acerca de la violencia que se produce en sus relaciones de pareja pero no se limita exclusivamente a la violencia que sufre la mujer sino que se analiza y valora el problema, independientemente de sexo y/o género tanto de víctimas como de agresores. El objetivo de este proyecto ha sido determinar la incidencia, tipología y grado de autopercepción para conocer y caracteriza el problema de cara a aportar propuestas para la prevención del mismo.

2. Violencia de género, violencia doméstica y violencia en las relaciones de pareja. Precisiones conceptuales

Más allá del fenómeno en cuanto a aspecto cuantitativos, nos interesa aclarar el sentido $y$ significado de distintos términos que se re fieren a la violencia que se ejerce contra la mujer, violencia de género, violencia doméstica violencia familiar, entre otros, los cuales vienen siendo utilizados frecuentemente como similares.

La raíz etimológica del término violencia remite al concepto de fuerza. Este sustantivo se corresponde con verbos tales como vio lentar, violar o forzar. A partir de esta primer aproximación semántica, podemos decir que la violencia es una forma de ejercicio del poder que se constituye en un recurso para la re solución de conflictos interpersonales y es utilizado para doblegar la voluntad de los demás (Corsi, 2001). La violencia, en sus distintas modalidades, pretende eliminar la oposición que se presenta ante el ejercicio del poder, me diante el control de la relación y por medio del uso de la fuerza.

El género es una construcción cultural, mientras que el sexo es una categoría que se refiere a los atributos biológicos y físicos di- ferenciales entre hombres y mujeres. La mayoría de los rasgos diferenciadores de ambos géneros son construcciones culturales, producto de la sociedad y no derivados necesariamente de la naturaleza (Colás, 2007). El gé nero se constituye como el resultado de un proceso de construcción histórica, social y cultural mediante el que se adjudican simbólicamente las expectativas y valores que cada sociedad atribuye a sus hombres y mujeres. El proceso de construcción de la persona no se realiza sin la determinación del género, devenimos sujetos generizados y, por lo tanto, la feminidad y la masculinidad son una construcción, consecuencia de hacer de una persona sexuada una mujer o un hombre (Mayobre, 2006). Como resultado de ese proceso de socialización y aprendizaje cultural unas y otros muestran los valores, atributos, roles, identidades y representaciones que le han sido asignados bajo la etiqueta del género.

Coincidimos con Maqueda (2006), cuando afirma que la explicación que se hace de la violencia ejercida contra las mujeres, en clave cultural y no biológica, es la que determina la perspectiva de género. Esta orientación ideológica que, no está generalizada, es claro indicador de la ausencia de una definición comúnmente aceptada. Por ello las distintas instituciones y los ordenamientos jurídicos acompañan siempre en su articulado una definición del concepto en función de los temas o aspectos que pretenden combatir. Por ejemplo, las Naciones Unidas en su Declaración sobre la Eliminación de la Violencia en contra de la Mujer de 20 de diciembre de 1993 entiende que ésta "constituye una manifestación de relaciones de poder históricamente desiguales entre el hombre y la mujer que han conducido a la dominación de la mujer $y$ a la discriminación en su contra por parte del hombre..." (Resolución 48/104 Asamblea General Naciones Unidas).

De la misma manera, la Resolución del Paramento Europeo sobre Tolerancia Cero ante la Violencia contra las Mujeres de 16 se sep- tiembre de 1977 la relaciona con el "desequilibrio en las relaciones de poder entre los se xos en los ámbitos social, económico, religioso o político".

A nivel estatal el artículo primero de la Ley Orgánica Española de Medidas de Protección Integral contra la Violencia de Género (LO $1 / 2004$ de 28 de diciembre) señala que el objeto de la Ley es "...actuar contra la violencia que, como manifestación de la discriminación la situación de desigualdad y las relaciones de poder de los hombres sobre las mujeres, se ejerce sobre éstas por parte de quienes sean o hayan sido sus cónyuges o de quienes estén o hayan estado ligados a ellas por relaciones de afectividad aun sin convivencia". Como vemos, esta Ley limita la violencia de género a aquella que se produce en el ámbito de la pareja pero obvia otras formas de violencia que se dan en la realidad de la mujer.

Es pertinente, pues, diferenciar y separa el término violencia de género de la violen cia familiar o violencia doméstica. No es lo mismo violencia de género que violencia do méstica o familiar porque la primera señala a la mujer al margen de lugares y las dos segundas ubican el problema, de forma exclusiva, en el ámbito familiar y, además, conno tan el espacio de lo doméstico a algo privado. Obviamente debe reconocerse que el medio familiar es un espacio propicio para el ejercicio de la violencia, pero también lo es la pareja y, sin embargo, ni uno ni otra agotan la multiplicidad en que puede manifestarse la violencia de género. Actualmente, y cada vez más de forma mayoritaria, se viene utilizando el término violencia de género el cual nos parece el más adecuado cuando nos referimos a aquellas formas de violencia basadas en el género y ejercida contra las mujeres (Ferrer 2006).

Nuestra investigación pretende aportar conocimiento acerca de la violencia que se produce en las relaciones de pareja que establecen jóvenes universitarios, analizando $y$ valorando el problema. Sabemos que este fe- 
nómeno trasciende y supera la violencia que se da de forma unidireccional del hombre hacia la mujer, por lo que no nos vamos a limitar a realizar un estudio sobe violencia de gé nero. Por esta razón hemos utilizado la etiqueta descriptiva violencia en las relaciones de pareja y ello porque el estudio que aquí se presenta se refiere a las relaciones violen tas que pueden darse en la pareja independientemente del lugar, sexo y/o género tanto de víctimas como de victimarios. En una primera aproximación, entendemos este término como un patrón regular de conductas violentas y coercitivas que, en el curso de las relaciones de pareja, y ejercidas tanto por el hombre como por la mujer, persiguen la consecución de una situación de conformidad y control sobre la víctima.

\section{Algunas investigaciones sobre la violen-} cia en las relaciones de pareja

La violencia que se da en las relaciones de pareja fuera del matrimonio ha sido muy poco estudiada. Está generalizada la idea de que debido al estrecho y continuo contacto que existe en las parejas casadas, pueden producirse más fácilmente situaciones de violencia y malos tratos que en el caso de parejas que no cohabitan. Efectivamente, tendemos a pensar que en las relaciones más abiertas existe menos compromiso y más libertad por lo que son menos proclives a que se produzcan situaciones que puedan generar conductas maltratantes; sin embargo, algunas revisiones (Barnet, Miller-Perrin y Perrin, 1997) indican que la incidencia de la violencia es más elevada que la que se da en la relación marital. En esta línea, otros estudios más recientes coinciden en el mismo argumento, Brownridge (2004) señala que un $7,1 \%$ de las parejas canadienses que cohabitaban habían sufrido violencia en los últimos cinco años frente al 3,2\% de de los matrimonios legalizados.

Así mismo, es habitual la idea de relacionar la violencia en las relaciones de pareja con personas de mediana y elevada edad; sin embargo, hoy sabemos que la violencia que se produce en las parejas de adolescente jóvenes es muy frecuente. Todas las investigaciones disponibles establecen que las re laciones violentas son un hecho significativo y repetitivo en las relaciones entre chicos y chicas jóvenes (Oliver y Valls, 2004). En la r visión llevada a cabo por Fuchs y Woessner (2004) sobre investigaciones realizadas en Europa, se concluye que las mujeres jóvenes son objeto de más violencia, por parte de sus parejas, que aqué llas de más edad.

También es frecuente asociar la violencia a personas con un bajo nivel de estudios. Straus (2004) en el estudio que, a nivel internacional, viene realizando con alumnado universitario de todo el mundo, señala que el $29 \%$ de los encuestados había cometido algún tipo de agresión hacia su pareja durante los doce meses anteriores. Kury, Obergfell-Fuchs y Woessner (2004) señalan que entre un 12,5\% un $28 \%$ de las estudiantes universitarias europeas son objeto de violencia en sus relaciones de pareja. Todos estos datos van en la misma línea que los aportados por otras investigaciones que han estudiado el mism tema (Charkow y Nelson, 2000; James et al., 2000; Smith, White y Holland, 2003). Como afirma Ferrer et al. (2006) un alto nivel de estudios no garantiza la presencia de actitude desfavorables hacia la violencia contra las mujeres en la pareja, de hecho en algunos estudios llevados a cabo con colectivos de profe sionales cualificados se han detectado actitudes favorables hacia esta forma de violencia.

Las investigaciones en el ámbito internacional corroboran que el fenómeno de la violencia en las relaciones de pareja también se da en el contexto universitario y que, en otros países, existen múltiples iniciativas y experiencias en universidades para prevenir la violencia de este tipo en ellas (Valls et al., 2007; Aguilar et al., 2009).

\section{Objetivos de la investigación}

El objetivo de esta investigación es realizar un estudio descriptivo del fenómeno de los malos tratos en las relaciones de pareja en el contexto de los estudiantes de magisterio en la Facultad de Ciencias de la Educación de la Universidad de Granada que nos ayude a caracterizar y a conocer el fenómeno en el ámbito específico universitario. Para ello pretendemos:

- Detectar la existencia de violencia en las relaciones de pareja.

- Cuantificar la incidencia de la violencia

en la pareja con relación a sus distintas tipologías.

- Determinar el grado de autopercepción de los malos tratos en las víctimas.

- Identificar las variables que en víctimas

puedan asociarse a la violencia en las relaciones de pareja.

- Elaborar propuestas educativas para la prevención de la violencia en las relaciones de pareja.

\section{Método}

\subsection{Muestra}

La población objeto de nuestro estudio ha estado constituida por el alumnado de $1^{\underline{0}}, 2^{\underline{0}}$ y $3^{0}$ curso que realiza estudios de magisterio en sus distintas especialidades (educación primaria, educación infantil, audición y lenguaje, educación especial, lengua extranjera, educación física y educación musical) en la Facultad de Ciencias de la Educación de la Universidad de Granada. La muestra quedó finalmente constituida por 493 estudiantes que respondieron al cuestionario entre los días 16 de abril y 11 de mayo de 2008 , de los cuales el $65,5 \%$ fueron mujeres $(n=323)$ y el $34,5 \%$ hombres $(n=170)$.

\subsection{Instrumento de recogida de datos}

Para dar respuesta a los objetivos planteados realizamos un estudio de carácter descriptivo, que intenta poner de manifiesto la existencia los tipos, la incidencia y el grado de autopercepción de la violencia en las relaciones de pareja en el colectivo señalado.

El instrumento, elaborado ex profeso para la realización de esta investigación, ha sido el Cuestionario para el estudio de la violencia en las relaciones de pareja (Soriano, 2006). Para su construcción se revisaron trabajos similares, entre otros, la Escala de severidad de violencia contra las mujeres (Marshall, 1992), Enquête nacional sur les violences envers les femmes en France (ENVEFF, 2000), Violencia contra las mujeres (Instituto de la Mujer, 2000), relacionados con nuestro estudio.

El instrumento se sometió a la valoración de profesionales que trabajan en el ámbito de la violencia de género y a expertos universitarios que trabajan la violencia en las relaciones de pareja. Tras la revisión y la valoración de los jueces y para aumentar su calidad téc nica se realizó un estudio piloto con un grupo de alumnos. La factorización de las respuestas confirmó la estructura del cuestionario de partida que finalmente fue utilizado como definitivo. Sus ítems ponen de manifiesto la violencia desde una triple perspectiva: la psico lógica, donde se pretende el dominio del otro a través de la desvalorización y la implantación de hábitos de sumisión; la sexual, en la que se pone de relieve el carácter sexual de la violencia que busca el control y la utilización del cuerpo de la pareja; y, por último, la física donde se muestra cómo el mecanismo de la agresión física trata de imponer el dominio por medio de la fuerza.

Sabemos que en la práctica las distintas formas en las que se manifiesta la violencia en las relaciones de pareja suelen darse asociadas quedando, muchas veces, difusas en los mismos comportamientos. Por este motivo nuestro cuestionario (Cronbach's Alpha 0250) las diferencia y separa para detecta más nítidamente su presencia y muestra acciones concretas en las que se presentan a tra- 
vés de una escala que indica la frecuencia de las mismas.

Dicho instrumento consta de diez categorías: maltrato físico, maltrato psicológico, abuso sexual, abuso económico, aislamiento social, desvalorización personal, control personal, abandono psicológico, desvalorización ideológica/religiosa y abuso por actividades domésticas, todas la cuales se explicitan por medio de veinte ítems. A través de la descripción de situaciones concretas, se pregunta directamente sobre la ocurrencia de tales comportamientos mediante una escala que contempla tres opciones de respuesta: nunca, alguna vez o con frecuencia. A partir de las respuestas será posible identificar al alumnado sometido a diferentes formas de violencia, independientemente de que sea o no consciente de ello.

\subsection{Recogida de datos e información}

Respecto a la recogida de datos, los cuestionarios fueron aplicados en horario de clases y se contó para ello con la participación de varios profesores que han formado parte de esta investigación. El alumnado fue informado adecuadamente del carácter voluntario y anónimo de su participación en el estudio as como de los objetivos del mismo.

Para realizar el análisis de los datos recogidos se ha utilizado el paquete estadístico SPSS versión 12.0. Dado el carácter descriptivo, exploratorio y explicativo del mismo, se han utilizado técnicas, desde el procesamiento de la información a nivel descriptivo (media, mediana, valor mínimo y máximo, desviación típica), hasta otras inferenciales de carácter paramétrico y no paramétrico que han ayudado a resaltar las relaciones entre las variables de la realidad estudiad.

\section{Análisis e interpretación de los resultados}

Con respecto a la incidencia de los malos tratos en las relaciones de pareja (véase tabla 1), diferenciando por géneros, el 73,3\% de las mujeres encuestadas señalan haber sido objeto de alguna de las formas de violencia señaladas en el cuestionario, mientras que el 21,1\% de los hombres ha sido objeto, por parte de su pareja, de alguna forma de violencia. Como se puede comprobar la diferencia entre hombres y mujeres es muy significativa con respecto a la incidencia que se produce.

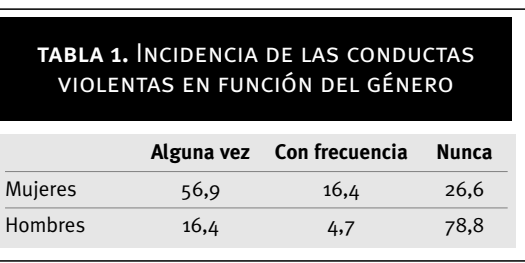

Con relación a la incidencia por tipologías, en el caso de la mujer (véase tabla 2) hemo obtenido los siguientes resultados:

\begin{tabular}{|lccc|}
\hline \multirow{3}{*}{$\begin{array}{l}\text { TABLA 2. INCIDENCIA DE LA VIOLENCIA SEGÚN } \\
\text { CATEGORÍAS (MUJERES) }\end{array}$} \\
\cline { 2 - 4 } Categorías & N & Mujeres \\
\hline Maltrato físico & 92,3 & 7 & CF \\
\hline Maltrato psicológico & 82,8 & 15,5 & 1,7 \\
\hline Abuso sexual & 98 & 2 & 0 \\
\hline Abuso económico & 89,3 & 8,7 & 2 \\
\hline Aislamiento social & 87,7 & 10,2 & 2,1 \\
\hline Desvalorización personal & 83,3 & 15,3 & 1,4 \\
\hline Control personal & 67,9 & 28,3 & 3,8 \\
\hline Abandono psicológico & 84 & 15 & 1 \\
\hline $\begin{array}{l}\text { Desvalorización } \\
\text { ideológico/religiosa }\end{array}$ & 85,6 & 12 & 2,4 \\
\hline $\begin{array}{l}\text { Abuso por actividades } \\
\text { domésticas }\end{array}$ & 82,5 & 15,8 & 1,7 \\
\hline
\end{tabular}

El maltrato psicológico es la categoría que con mayor frecuencia se da. De las mujere que han señalado sufrir comportamientos violentos, todas marcaron alguna de las distintas subcategorías del maltrato psicológico. Entre todas ellas la que mayor incidencia presenta es el control personal que es señalado en el $32,1 \%$ de los casos.

El maltrato físico es la categoría que ocupa el segundo lugar y es sufrida por el $7,7 \%$ del alumnado femenino. Se da a veces en 7 de cada 100 casos y con frecuencia en el o, $7 \%$.

En tercer lugar, la categoría abusos sexuales ha sido señalada a veces por 2 de cada 100 alumnas encuestadas y en ningún caso las conductas abusivas se han producido con frecuencia.

Con respecto a los hombres, señalamos que el $21,1 \%$ del alumnado que ha participado en la investigación, ha padecido, por parte de su pareja, alguna vez o con frecuencia alguna de las formas de malos tratos de las señaladas en los distintos ítems del cuestionario, el $4,7 \%$ asegura haber sufrido alguna de esas conductas con frecuencia y el $16,4 \%$ alguna vez (véase tabla 3).

Por categorías, podemos destacar los siguientes resultados:

El maltrato psicológico, al igual que ocurre con las mujeres, es la categoría que ma-

\begin{tabular}{|c|c|c|c|}
\hline \multicolumn{4}{|c|}{$\begin{array}{l}\text { TABLA 3. INCIDENCIA DE LA VIOLENCIA SEGÚN } \\
\text { CATEGORÍAS (HOMBRES) }\end{array}$} \\
\hline \multirow[t]{2}{*}{ Categorías } & \multicolumn{3}{|c|}{ Hombres } \\
\hline & $\mathbf{N}$ & AV & CF \\
\hline Maltrato físico & 94,2 & 5,5 & 0,3 \\
\hline Maltrato psicológico & 89,7 & 9,1 & 1,2 \\
\hline Abuso sexual & 99,4 & 0,6 & o \\
\hline Abuso económico & 93,7 & 5,4 & 0,9 \\
\hline Aislamiento social & 90 & 9,1 & 0,9 \\
\hline Desvalorización personal & 89,3 & 9,8 & 0,9 \\
\hline Control personal & 82,1 & 15,9 & 2 \\
\hline Abandono psicológico & 87,3 & 12,1 & 0,6 \\
\hline $\begin{array}{l}\text { Desvalorización } \\
\text { ideológico/religiosa }\end{array}$ & 91,8 & 7,3 & 0,9 \\
\hline $\begin{array}{l}\text { Abuso por actividades } \\
\text { domésticas }\end{array}$ & 87,1 & 12 & 0,9 \\
\hline
\end{tabular}

yor incidencia presenta. Del total de hombres que señalaron sufrir comportamientos violentos, en todos los casos, marcaron alguna de las distintas formas del maltrato psicológico. De entre todas ellas la que con mayor frecuencia se da es el control personal que presenta una incidencia del 16,1\%. En esta cate goría la incidencia que se da es de 16 puntos por debajo de las mujeres.

$\mathrm{Al}$ igual que en el caso de sus compañera de estudios, el maltrato físico es la categoría que ocupa el segundo lugar, y es señalada por el $5,8 \%$ del alumnado masculino. Se da a veces en 5,5 de cada 100 casos y con frecuencia en el $0,3 \%$, (respectivamente 1,5 y o,4 puntos menos que en el caso de sus compañeras).

Los abusos sexuales, tercera categoría en orden de incidencia, se ha producido a veces en o,6 de cada 100 alumnos encuestados y en ningún caso con frecuencia.

Con respecto al tercer objetivo planteado en la investigación, determinar el grado de au topercepción de los malos tratos en las víctimas, sólo el 2,6\% de las alumnas se conside ran objeto de malos tratos por parte de su pareja. Este dato nos revela que un importante número de alumnas sufre malos tratos sin se conscientes de ello, concretamente un $97,4 \%$.

En cuanto a la autopercepción que de los malos recibidos tienen los alumnos encuestados, tan sólo un $1,2 \%$ se consideran maltratados por parte de su pareja por lo que el $98,8 \%$ sufre estas situaciones sin ser conscientes de ello lo cual nos revela el elevadísimo número que sufre violencia en su relación de pareja sin saberlo

\section{Conclusiones}

Todos los datos anteriores nos llevan a afirma que la incidencia de las distintas conductas violentas a que han sido sometidas las alumnas por parte de su pareja es muy alta y, por tanto, especialmente preocupante, el 73,3\% marcaron uno o más ítems a veces o con fre cuencia, y el 16,4\% marcó uno o más ítems con frecuencia. Igualmente, aunque con una incidencia significativamente más baja que en el caso de sus compañeras $(52,2$ puntos por 
debajo en el total y 11,7 puntos en el parcial con frecuencia), los alumnos también sufren comportamientos maltratantes por parte de sus parejas, el 21,1\% marcaron uno o más ítems a veces o con frecuencia y el 4,7\% marcó uno o más ítems con frecuencia.

En todas las categorías los hombres son objeto de menos comportamientos violentos por parte de su pareja que en el caso de las mujeres. La diferencia en cuanto a la presencia de las distintas conductas oscila entre los 14,2 puntos que se da en el control personal y las 1,4 décimas del abuso sexual, señalando no obstante que en el caso de los alumnos, esa conducta abusiva se presenta, en todos los casos señalados, en relaciones establecidas con parejas homosexuales. Las investigaciones realizadas sobre la violencia ejercida contra los hombres en sus relaciones de pareja, coinciden en señalar que la violencia ejercida contra ellos es llevada a cabo, en muchas ocasiones, por otros hombres (Oliver y Valls, 2004).

Todos estos datos indican que el índice de violencia existente en las relaciones de pareja de nuestro alumnado es muy alto. Con relación al género de las personas maltratadas, los datos que aportan los estudios existentes en nuestro país (Alberdi y Matas, 2000; Instituto de la Mujer, 2000; González Méndez y
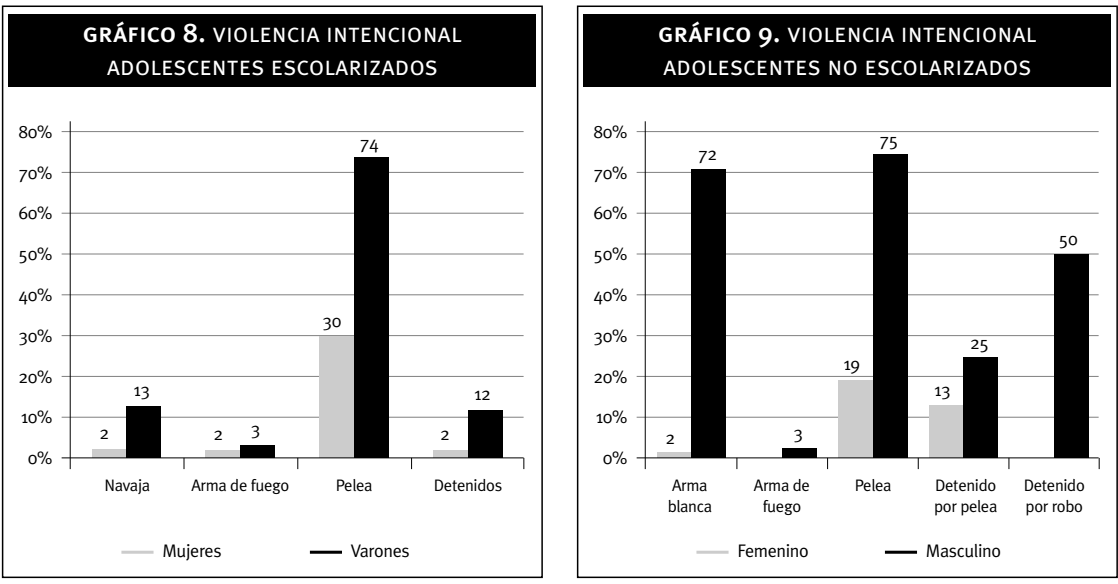

las víctimas ni por los agresores pues, generalmente, se confunde maltrato y ofensas con amor e interés por la pareja. Creemos que ésta es una de las razones por las que las parejas no rompen su relación. Esta normalización de la violencia que se produce en los patrones de convivencia es una de las causas de que se introyecte, se asuma y se reproduzca. Para reaccionar ante este problema es imprescindible ser conscientes de su existencia pero, como hemos visto, solamente un mínimo porcentaje de las mujeres y de los hombres encuestados reconocen su situación de malos tratos, para el resto, que es la gran mayoría, este problema es invisible.

Este problema afecta a la sociedad en su conjunto, siendo muchas las mujeres jóvenes, adultas y mayores que, también con buen nivel de estudios y formación, entran en un proceso de degradación personal y social que va unido a su pareja, la cual de una forma natural y socialmente aceptada, impone de forma sistemática su voluntad. La aceptación de la violencia trasciende los estereotipos culturales, las clases sociales y, además, como vemos, no se supera con una mayor formación académica. Como dice Straus, (2004), incluso para los grupos de personas con un alto nivel educativo, como son los estudiantes universitarios, hay un largo camino que recorrer para cambiar las normas culturales que toleran la violencia en las relaciones de pareja.

Todos los datos anteriores son realmente preocupantes porque denotan claramente la falta de información y el bajo nivel formativo que, con respecto a este problema, presenta nuestro alumnado. En general, carecen de conocimientos específicos acerca de qué es la violencia y los malos tratos y difícilmente se puede reaccionar ante un problema si éste no es visible a los ojos de los implicados, además ciertos comportamientos violentos están tan arraigados culturalmente que no es fácil identificarlos y sólo el reconocimiento como tales nos permitirá visualizar el problema. Pero si este asunto es ya grave en sí mismo, adquiere, si cabe, tintes más preocupantes cuando estos datos se refieren a futuros maestros, por ello aunque trabajar en la detección y prevención de la violencia en las relaciones de pareja es responsabilidad de muchos lo es especialmente de los profesionales que, como en nuestro caso estamos encargados de la formación Eloblema de la violencia en la pareja ha permanecido oculto y ausente como tema de estudio en la formación curricular de estos futuros profesionales, por ello el currículum formativo destinado a nuestros alumnos debería dedicar un espacio específico al conocimiento y a la reflexión sobre las relaciones de poder, la violencia en general, y los malos tratos en las relaciones de pareja, en particular. No basta con formar en contenidos teóricos y en estrategias para trabajar en el aula actividades no sexistas, sino que se ha de promover la toma de conciencia acerca de cómo influyen nuestros propios comportamientos docentes. Todo ese conocimiento debe ser generado a través de la crí tica y de la reflexión para ser aplicado y, por tanto, actuar como fuerza transformadora de la realidad.

Finalmente presentamos algunas propuestas más concretas para trabajar en la formación y prevención de la violencia en las relaciones de pareja en el ámbito universitario:

- Inclusión en el currículum formativo de alumnado de las Facultades de Educación de contenidos relativos a la violencia, especialmente la que se da en las relaciones de pareja.

- Inclusión en el currículum formativo de alumnado que realiza el Master de Educación Secundaria de estos mismos contenidos, puesto que, en su gran mayoría serán profesores de formación profesio nal, educación secundaria y bachillerato. - Diseño de materiales específicos para la impartición de talleres sobre la violencia en las relaciones de pareja dirigido a todo 
el colectivo universitario donde se trabajen los siguientes objetivos:

- Fomentar la reflexión crítica sobre el género, la igualdad, la violencia y las relaciones de pareja.

- Analizar y concienciar al alumnado sobre la importancia de la educación de lo sentimental y de las emociones como forma de desarrollo integral libre de prejuicios y estereotipos de género.

Proporcionar al alumnado imágenes alternativas ofreciendo modelos de hombre y de mujer no asociados a comportamientos violentos.

Concienciar al alumnado sobre la gravedad de las consecuencias personales y sociales de la violencia en las relaciones de pareja.

- Analizar y construir colectivamente valores acordes con la resolución no violenta de conflictos en las relaciones de pareja.

Por último, creemos necesario insistir en la idea de que la educación en la igualdad entre hombres y mujeres y la socialización en la resolución pacífica de conflictos son los pilares básicos sobre los que sustentar la prevención de la violencia. Para ello es necesario llevar a cabo una educación de los géneros, mediante un currículum abierto, teniendo en cuenta las desigualdades o atributos distintos de una realidad social integrada por hombres y mujeres. Éste es el primer paso para poder llevar a cabo una educación basada en la no discriminación y en una igualdad que sea respetuosa con la diferencia. A toda esta tarea debe contribuir la educación, desde la educación infantil hasta los estudios universitarios, estamos hablando, por tanto, de una educación a lo largo de la vida y para la vida.

\section{Referencias bibliográficas}

Aguilar, C. et al (2009): "Violencia de género en el ámbito universitario. Medidas para su superación" Pedagooía Social Revista Interuniversitaria 85-94

Alberdi, I. y Matas, N. (2000): La violencia doméstica. Informe sobre los malos tratos a mujeres en España. Fundación. Barcelona: La Caixa.

Barnet, O. W., Miller-Perrin, C.L y Perrin, R. (1997) Family violence across the lifespan. Londres: Sage Brownridge, D.A. (2004): "Understanding Women's heightened risk of violence in Common-Law Unions. Revisting the selection and relationship hypotheses". Violence Against Women, 10:6, pp 626-65

Charkow, W, y Nelson, E. (2000): "Relationship de pendency, dating violence and scripts of female college student". Journal of College Counseling, 3: 1, pp. 12-17.

Cobo, J.A. (2003):

Cttp:/wwwinicia.es/de/VictorArrogante/Archivos de_Principal/ Holmbres_Mujeres_Igualdad/ La_violencia_con_historia.m (fecha de acceso: marzo 2008)

Colás, P. (2007): “La construcción de la identidad de género: enfoques teóricos para fundamentar la investigación intervención palua fivar. $R$ Investigación Educativa, 25/1, pp. 151-156.

Corsi, J. (2001): Violencia familiar. Una mirada in terdisciplinaria sobre un grave problema social. Buenos Aires: Paidós.

Echeburúa, E. y de Corral, P. (1998): Manual de vio lencia familiar. Madrid: Siglo XXI de España Edi-

ENVEFF (2000): Enquête nacional sur les violences envers les femmes en France. París: Service des Droits des femmes.

Ferrer, V.A. et al (2006): “¿De qué hablamos cuando hablamos de violencia de género?" en Esteve, J. M. y Vera, J. (Coord), Educación Social e igualdad de genero. Málaga: Ayuntamiento de Málaga, pp. 209231.

Ferrer, V.A. et al (2006): "La violencia contra las mujeres en la pareja: creencias y actitudes en estu- diantes universitarios/as". Revista Psicothema, 18/3, pp. 359-366.

González Méndez, R. y Santana, J.D. (2001): "La violencia en parejas jóvenes". Revista Psicothema. 13/1, pp. 127-131.

Instituto de la Mujer (2000): La violencia contra las mujeres. Resultados de la macroencuesta. Madrid: Ministerio de Trabajo y Asuntos Sociales.

James, W.H., West, C., Deters, K.E., y Armijo, E. (2000): "Dating violence". Adolescence, 35: 139, pp. $455-466$.

Kury, H., Obergfell-Fuchs, J. y Woessner, G. (2004): "The Extent of Family Violence in Europe. A com paraison of National Surveys". Violence Agaisn Women, 10/7, pp. 749-769.

ey Orgánica 1/2004, de 28 de diciembre, de Me didas de Protección Integral contra la Violencia de Género, BOE n. ${ }^{\circ} 313$ de 29 diciembre del 2004.

Maqueda, M.L. (2006): La violencia de género. Entre el concepto jurídico y la realidad social.

Revista electrónica de Ciencia Penal y Criminología, 8-2, pp. 1-13

http://www.juridicas.unam.mx/sisjur/familia/pdf/ 15-189s.pdf (fecha de acceso: mayo 2008)

Marshall L. (1992): "Development of the severity of violence again lence, 7, pp.103-121.

Mayobre, P. (2006): “La formación de la identidad de género. Una mirada desde la filosofía", en Esteve, J. M. y Vera, J. (Coord), Educación Social e igualdad de género. Málaga: Ayuntamiento de Málaga, pp. 21-54

Naciones Unidas (1993): Resolución 48/104 de la Asamblea General de las Naciones Unidas.

Naciones Unidas (2003): Declaración de las Naciones Unidas sobre la eliminación de la violencia contra la mujer 48/104 de 20 de diciembre del 2003.

Oliver, E. y Valls, R. (2004): Violencia de género. Investigaciones sobre quienes, por qué y cómo superarla. Barcelona: El Roure.

Parlamento europeo (1997): Resolución de 17 de septiembre de 1997 sobre la Campaña Europea de Tolerancia Cero.

Smith, P.H., White, J.W., y Holland, L. (2003): "A

ongitudinal perspective on dating violence among adolescent and college-age women". American Journal of Public Health, 93:7, pp. 104-110.

Soriano, A. (2006): Cuestionario para la detección de la violencia en las relaciones de pareja. Granada: Registro $\mathrm{n}^{\mathrm{Q}}$ GR $2760 / 2006$.

Strauss, M. (2004): Prevalence of violence agains dating partners by male and female university students worldwide. Violence Against Women 10/7, pp. 790-811.

Valls, R. et al (2007): “¿Violencia de género también en las universidades? Investigaciones al respecto". $R$ vista de Investigación Educativa, 25/1, pp. 219-231.

\section{Notas}

${ }^{1}$ Plan Propio de Investigación de la Universidad de Granda. Referencia del proyecto: 5776/11/11/2006

DIRECCIÓN DEL AUTOR: Andrés Soriano Díaz. Facultad de Ciencias de la Educación. Universidad de Granada. Campus Universitario “La Cartuja, s/n" 18071 Granada

Correo electrónico: asoriano@ugr.e

Fecha de recepción del artículo: 09.XII.2009 Fecha de revisión del artículo: 09.XII.2009 Fecha de aceptación definitiva: 19.II.2010

\section{COMO CITAR ESTE ARTíCULO:}

Soriano Díaz, A. "La violencia en las relaciones de pareja en estudiantes universitarios. Propuestas educativas" Pedagogía Social. Revista Interuniver sitaria. 18. pp. 87-97 